Z. klin. Chem. u. klin. Biochem.

8. Jg., S. $602-604$, November 1970

\title{
Nachweis, Isolierung und Charakterisierung von Peptiden in Ductus thoracicus-Lymphe ${ }^{1}$ )
}

\author{
Von K. Schumacher, U. Wehmer, W. Koch, W. Schneider und Th. Georghiu
}

Mediquinische Universitätsklinik Köln (Direktor: Prof. Dr. R. Gross) und Marien-Krankenbaus Hamburg, Cbirurgische Abteilung (Chefarzt: Prof. Dr. H. Scbreiber)

(Eingegangen am 17. Juli 1970)

Im Lymphserum von Patienten mit Lebercirrhose wurden 3 Peptide, davon ein Glycopeptid, in hoher Konzentration nachgewiesen. Das Molekulargewicht des Glycopeptids wurde zu etwa 1600 bestimmt. Die Aminosäure-Analyse der Peptide ergab nur saure und neutrale Aminosäuren. Entsprechend dem Fehlen von basischen Aminosäuren war auch eine Aggregation von Thrombocyten nicht auslösbat. Die gaschromatographische Analyse des Glycopeptids ergab eine Reihe von Zuckerkomponenten. Über eine mögliche biologische Bedeutung dieser Peptide bei Patienten mit Lebercirrhose kann keine Aussage gemacht werden.

\section{Demonstration, isolation and characterisation of peptides in lymph from the thoracic duct}

Three different peptides, including a glycopeptide, were found in high concentration in the lymph serum from the lymph of the thoracic duct of patients with liver cirthosis. The estimated molecular weight of the glycoprotein was 1600 . Only anionic and neutral amino acids could be detected in the peptides, which, owing to the absence of cationic amino acids, would not induce the aggregation of blood platelets. Gas chromatographic analysis of the glycopeptide showed many different sugars. The biological significance of these peptides is unknown.

Bei Untersuchungen über das Proteinmuster der Ductus thoracicus-Lymphe von Patienten mit Lebercirrhose (1) konnten wir in einigen Lymphseren eine niedermolekulare Fraktion nachweisen, die im Blutserum derselben Patienten nur in kleiner Menge vorhanden war. Im nachfolgenden wird die niedermolekulare Fraktion aus Lymphserum beschrieben.

\section{Material und Methoden}

Lymphe wurde nach Kanülierung des Ductus thoracicus während des Anlegens einer lymphovenösen Anastomose entnommen. Nach spontaner Gerinnung wurde das Lymphserum durch Abzentrifugieren der Zellbestandteile gewonnen.

Die präparative Fraktionierung der Lymph- und Blutseren erfolgte durch Molekularsiebung an Sephadex G-200 in 0,1M Tris HCl-Puffer, $\mathrm{pH} 8,0$ mit Zusatz von $0,1 \mathrm{M} \mathrm{NaCl}$ (2). Zur weiteren Reinigung der Peptide wurde eine Gelfiltration an Sephadex $\mathrm{G}-25$ in $\mathrm{H}_{2} \mathrm{O}$ angeschlossen.

Zur analytischen Trennung der Lymphproteine wurde die Elektrophorese im Polyacrylamidgel wie früher beschrieben $(3,4)$ durchgeführt. Die Aminosäure-Analyse der Peptide wurde nach der Vorschrift von MOORE und STEIN (5) mit einem automatischen Aminosäure-Analysator (Beckman Instr., Spinco Division, California) durchgeführt ${ }^{2}$ ).

Die Analyse der Kohlenhydratkomponenten der Peptide wurde gaschromatographisch durchgeführt (6). Salzfreies Lyophilisat wurde in $3 \mathrm{~N} \mathrm{HCl}$ bei $100^{\circ}$ für $3 \mathrm{Stdn}$. in versiegelten DickwandAmpullen unter $\mathrm{N}_{2}$ nach dem früher beschriebenen Verfahren (7) hydrolysiert. Nach Silylierung mit Hexamethyldisilazan und Trimethylcblorsilan im Pyridin-Milieu wurden $0,5 \mu l(0,25 \mu \mathrm{g})$ der hexangelösten per-O-Trimethylsilyl-Zuckerderivate auf einer polaren Säule (EGS 18\%/Chromosorb W) bei konstanten, programmierten Temperaturverhältnissen gaschromatographisch mit dem Gerät der Fa. Packard, Instr. GmbH, Frankfurt, mit Argon${ }^{3}$ H-Ionisations- bzw. Flammen-Ionisations-Detektor analysiert.

1) Mit Unterstützung der Deutschen Forschungsgemeinschaft. 2) Für die Durchführung der Aminosäure-Analyse danken wir Herrn Professor Dr. D. FASOLD, Physiologisch-chemisches
Institut Würzburg.
Die Bestimmung erfolgte unter Einbeziehung eines internen Standards (L-Fucose).

Die Bestimmung der Aggregation von humanen Thrombocyten wurde nach einer früher beschriebenen Methode (8) mit Hilfe eines automatischen Partikel-Zählgerätes (Coulter Electronics Itd. England, Modell A) durchgeführt.

\section{Ergebnisse}

Die Fraktionierung von Lymphseren von Patienten mit Lebercirrhose durch Molekularsiebung an Sephadex G-200 (Abb. 1a) ergab gegenüber der Fraktionierung

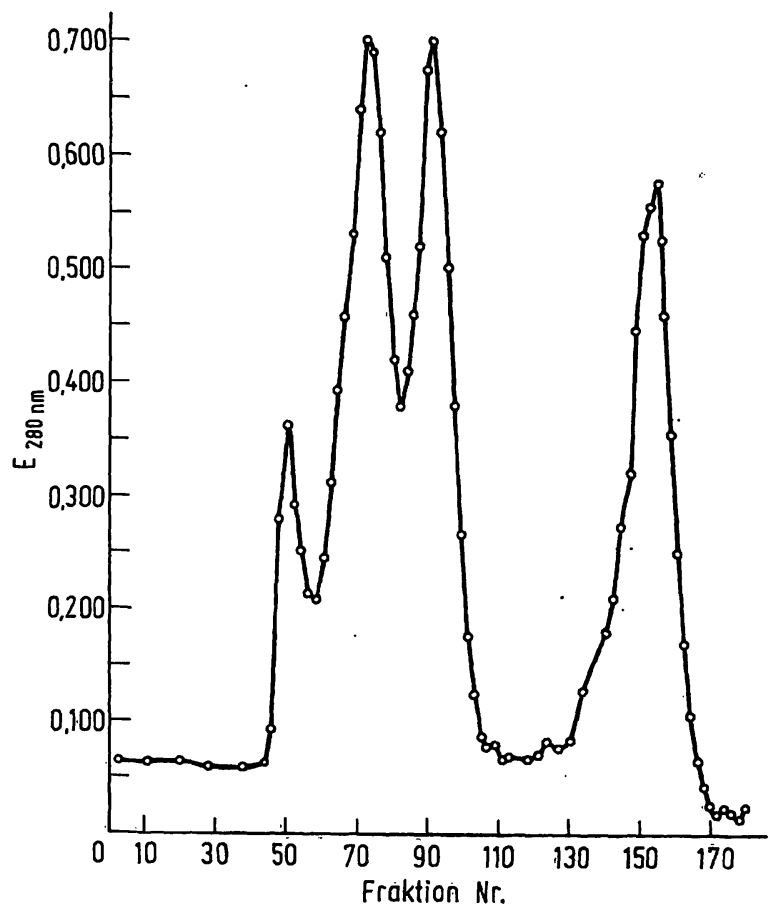

Abb. 1 a

Fraktionierung von Lymphserum eines Patienten mit Lebercirrhose an Sephadex G-200 
von normalem Lymphserum (Abb.1b) deutliche Abweichungen. Neben der relativen Vermehrung der $7 \mathrm{~S}$ Proteine fand sich in Lymphseren von Patienten mit Lebercirrhose eine niedermolekulare Fraktion in relativ großer Menge. Diese Fraktion war auch in normalem Lymphserum und im Blutserum von gesunden Probanden und von Patienten mit Lebercirrhose nachweisbar, jedoch nur in geringer Konzentration.

Nach Dialyse des Lymphserums war die niedermolekulare Fraktion nicht mehr nachweisbar.

Die weitere Fraktionierung der niedermolekularen Fraktion an Sephadex G-25 ergab eine Auftrennung in 3 Unterfraktionen (Abb. 2). Die Elutionsvolumina dieser Unterfraktionen waren nur wenig unterschiedlich. Entsprechend dem Elutionsvolumen an einer geeichten

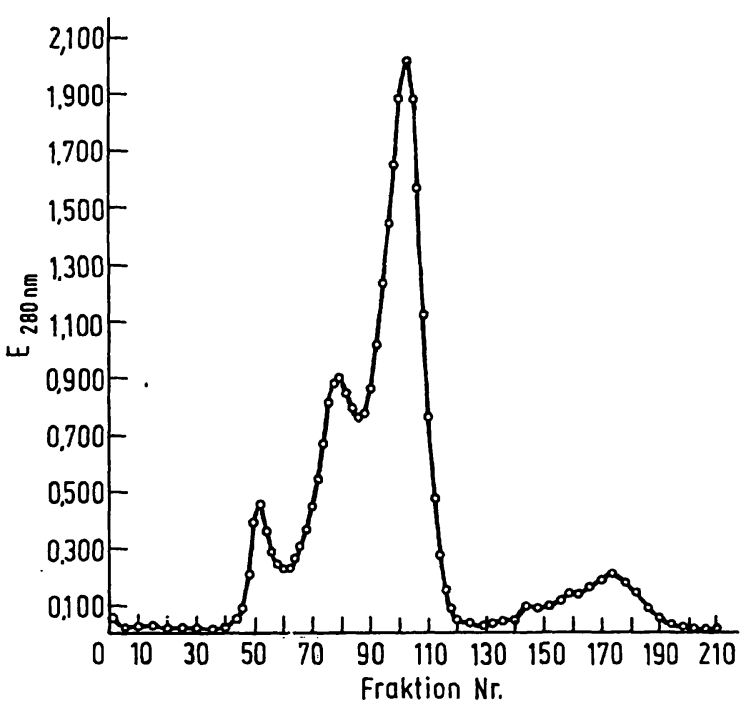

Abb. 1b

Fraktionierung von normalem Lymphserum an Sephadex G-200

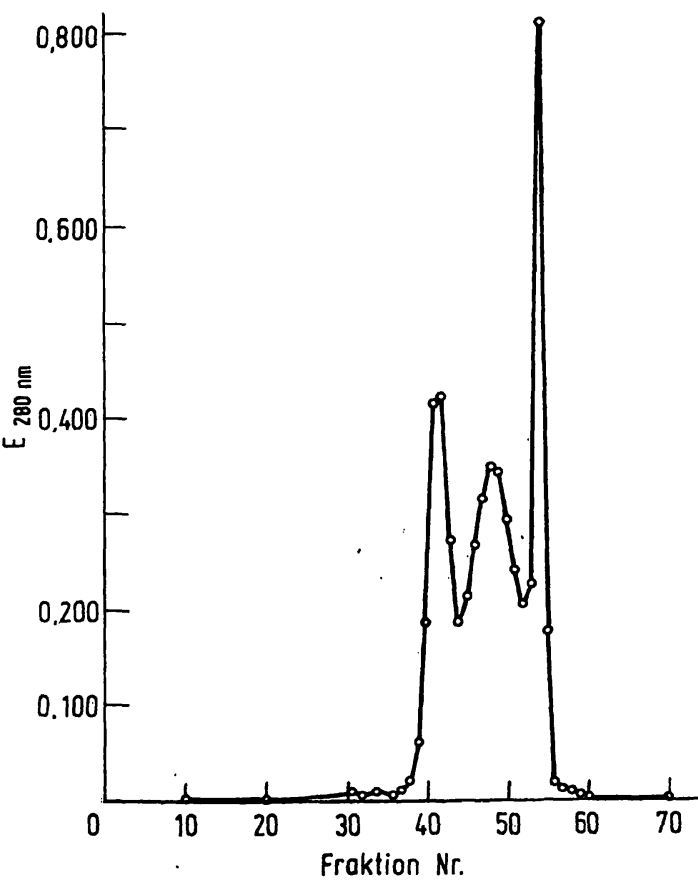

Abb. 2

Fraktionierung der isolierten Peptide an Sephadex 0-25
Gelsäule wurde für die mittlere Unterfraktion ein ungefähres Molekulargewicht von 1600 bestimmt.

Durch Elektrophorese im Polyacrylamidgel ließen sich die Unterfraktionen nicht trennen. Sie ergaben eine homogene, wenn auch etwas breite Bande (Abb. 3).

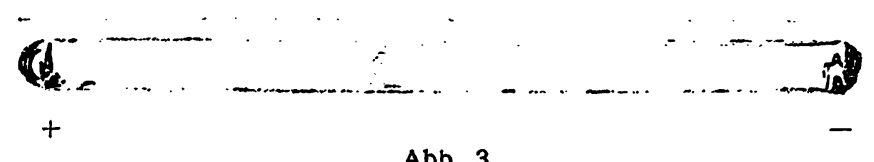

Polyacrylamidelektrophorese der Fraktion IV nach Gelfiltration von Lymphserum an Sephadex G-200

Immunologisch waren die niedermolekularen Fraktionen nicht nachweisbar, jedenfalls fanden wir immunelektrophoretisch nach Diffusion gegen Antihumanseren und gegen monospezifische Antiseren, einschließlich AntiBenCE-Jones-Serum keine Präzipitationsbande.

Die Aminosäureanalyse der 3 Unterfraktionen ergab nur saure und neutrale, jedoch keine basischen Aminosäuren (Tab. 1).

Tab. 1

Aminosäureanalyse der Fraktion II nach Gelfiltration der niedermolekularen Fraktion an Sephadex G-25

\begin{tabular}{cc}
\hline Aminosäure & $\mu$ Mol/Ansatz \\
\hline Asp & 0,017 \\
Thr & 0,030 \\
Ser & 0,030 \\
Glu & 0,096 \\
Pro & Spur \\
Gly & 0,162 \\
Ala & 0,278 \\
Val & 0,071 \\
Ile & Spur \\
Leu & 0,054 \\
His & Spur \\
\hline
\end{tabular}

Der Aminosäure-Zusammensetzung entsprechend führten die Fraktionen auch nicht zur Aggregation von Thrombocyten, wie dies von basischen (kationischen) Polypeptiden bekannt ist (8).

Die gaschromatische Analyse der 3 Unterfraktionen ergab nur in der 2. Unterfraktion verschiedene Kohlenhydrate (Abb. 4). In dieser Fraktion fanden wir $\alpha$-Mannose, $\alpha$-Glucose, $\alpha$-Galaktose, $\alpha$-Glucosamin, $\alpha$-Galaktosamin, $\beta$-Mannose, $\beta$-Glucosamin, $\beta$-Galaktosamin, $\beta$-Glucose und Acetyl-Hexosamin. Die erste und dritte Fraktion enthielten keine Kohlenhydratanteile.

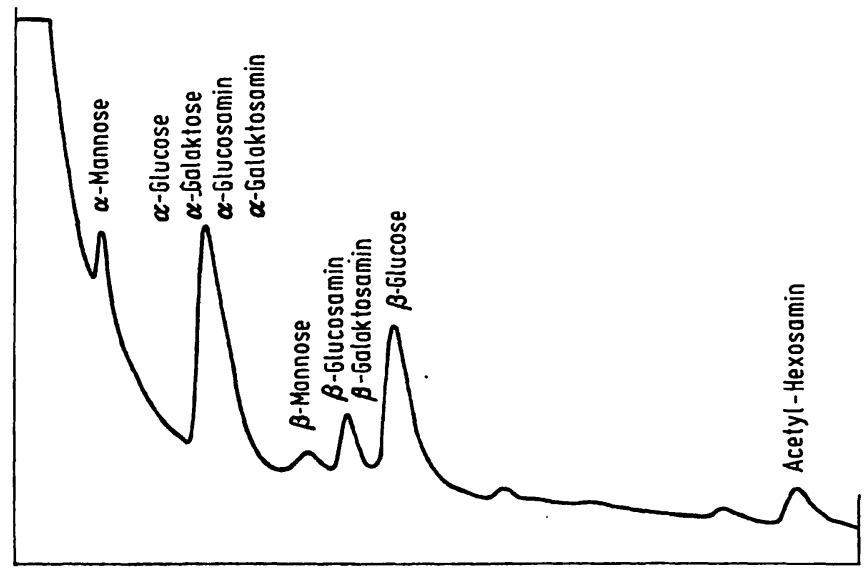

Abb. 4

Gaschromatographische Analyse des Glycopeptids (Fraktion II nach Gelfiltration an Sephadex G-25) aus Lymphserum 


\section{Diskussion}

Das Lymphserum von Patienten mit Lebercirrhose unterscheidet sich von normalem Lymphserum in verschiedener Hinsicht. Die Protein-Konzentration pathologischer Lymphseren zeigt starke Schwankungen, liegt aber im Mittel etwa in der gleichen Größenordnung wie die von normalen Lymphseren. Neben dem für Lymphseren offenbar typischen geringen Gehalt an IgM fanden sich in pathologischen Lymphseren eine relativ deutliche Vermehrung von IgA, ein lebertypisches Muster der Isoenzyme von Lactatdehydrogenase und teilweise lösliche Leberproteine (1).

Der auffallendste Unterschied zwischen normalen und pathologischen Lymphseren war die hohe Konzentration einer auch in normalen Lymphseren und in Blutseren nachweisbaren niedermolekularen Fraktion. Nach Isolierung konnte die zweite Unterfraktion als ein Glycopeptid mit einem Molekulargewicht von etwa 1600 charakterisiert werden. Dieses Glycopeptid erwies sich in der Polyacrylamid-Elektrophorese als homogen. Basische Aminosäuren waren in allen 3 Peptiden nicht nachweisbar. Demzufolge waren sie auch nicht in der Lage, eine Aggregation von Thrombocyten herbeizuführen.

Nach der Aminosäure-Zusammensetzung der Peptide ist eine Beziehung zum Kinin-System auszuschließen (9).
Die Testung einer möglichen Gefäßwirksamkeit verlief ohne Ergebnis (9).

Die Herkunft der Peptide im Lymphserum ist unbekannt. Sicher ist, daß diese Peptide nicht aus dem Blutserum stammen, denn sie waren im Blutserum der jeweils gleichen. Patienten nur in geringer Konzentration nachweisbar. Man muß annehmen, daß diese Peptide aus dem Quellgebiet dér Lymphe, dem Darm oder der Leber in die Lymphe und damit in das Blut gelangen.

Die Konzentration dieser Peptide in der Lymphe war nur bei 4 von 7 Patienten mit Lebercirrhose gegenüber dem Blutserum in dem hier beschriebenen Ausmaß erhöht. Eine Zuordnung der Vermehrung dieser Peptide zu unterschiedlichen Begleitkrankheiten bzw. Komplikationen der Lebercirrhose (Blutung aus Ösophagusvarizen oder Ascites) war nicht möglich. Hohe Konzentrationen der Peptide fanden wir gleich häufig bei $\mathrm{Pa}$ tienten mit und ohne Ascites. Demnach ist eine funktionelle Bedeutung der Peptide im Sinne einer Hemmung oder Steigerung der Ascitesbildung ähnlich der Wirkung von Adrenalin oder Histamin und Acetylcholin unwahrscheinlich.

Über eine mögliche biologische Bedeutung der in Lymphseren nachgewiesenen Peptide bzw. Glycopeptide können wir demnach keine Aussage machen.

\section{Literatur}

1. Schumacher, K., U. WehMier, W. Koch, W. Schneider und R. Gross, Klin. Wschr. (im Druck). - 2. Schumacher, K., Klin. Wischr. 45, 1045 (1967). - 3. Felgenhauer, K., S. BACH und A. Stannirer, Klin. Wschr. 45, 371 (1967). - 4. Schumacher, K., W. SCHNEIDER und R. Gross, Thrombos. Diathes. haemorrh. Stuttg. $X i X, 430$ (1968). - 5. Moore, St. und W. H. Sted, J. biol. Chemistry 176, 367 (1948). - 6. Georghiu, Th. und K. Oetre, J. Chromatogr. 48, 34 (1970). - 7. GeOrghiv, Th., K. Oette und V. BaumanN, Z. Naturforsch. 256,829 (1970). - 8. SCHNEIDER, W., W. KüBler und R. Gross, Thrombos. Diathes. haemorrh. (Stuttg.) $X X, 314$ (1968). - 9. HabermanN, E., Persönliche Mitteilung.
Priv. Doz. Dr. K. Schumacher Med. Univ. Klinik $5000 \mathrm{Köln}$-Lindenthal 


\section{Im Zeichen des Einhorns}

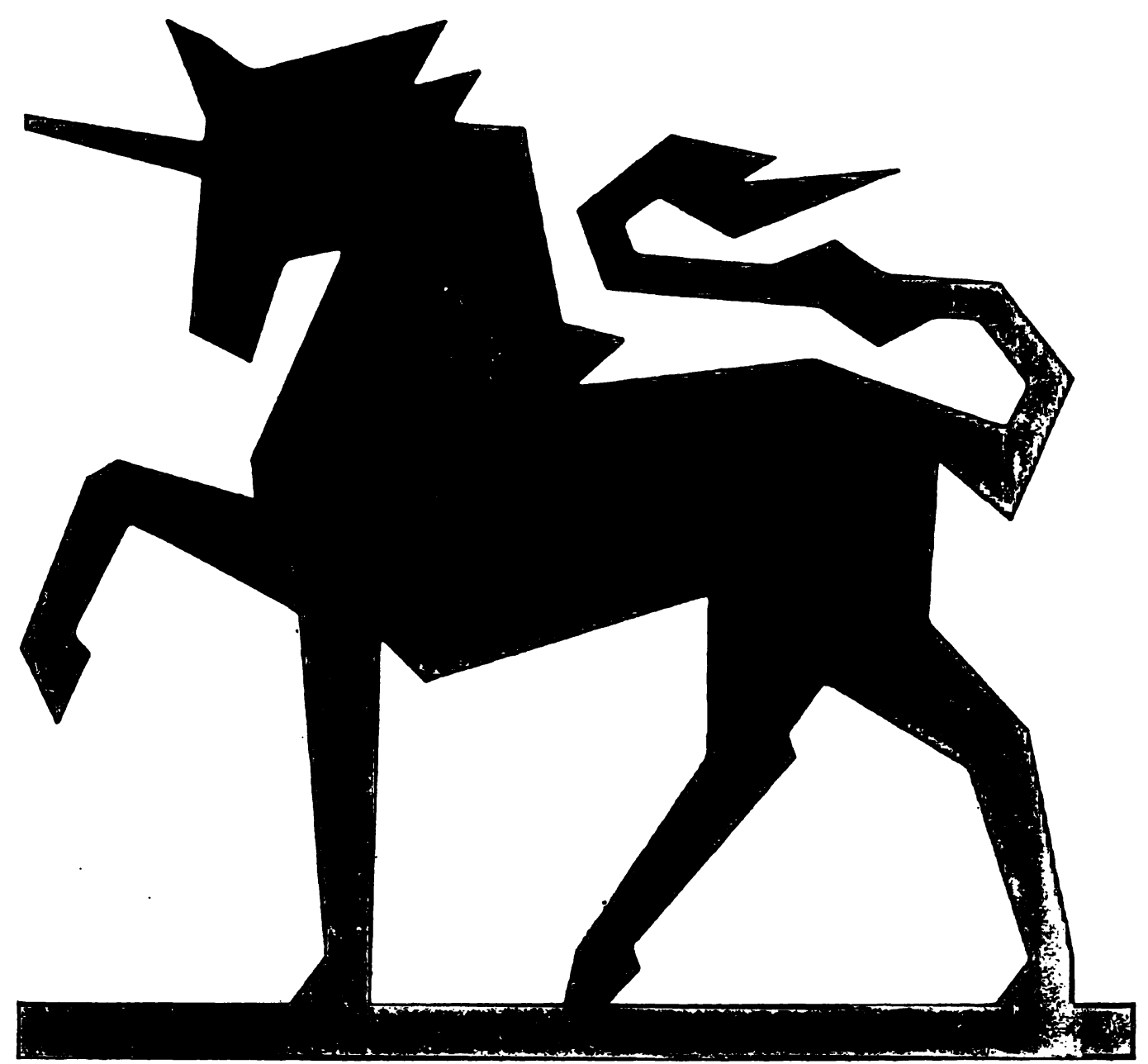

Wellcome Reagenzien

Qualitätsprodukte.

für das serologische

und bakteriologische

Laboratorium.

Hersteller:

Vertrieb

Wellcome Reagents Ltd.

Wellcome Research Laboratories England

für die Bundesrepublik:

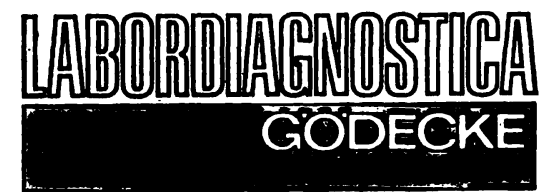




\section{WME HSLTEN SIE ES \\ MUT OER ROUTINEARBEIT? BESGE: IE HALTEN

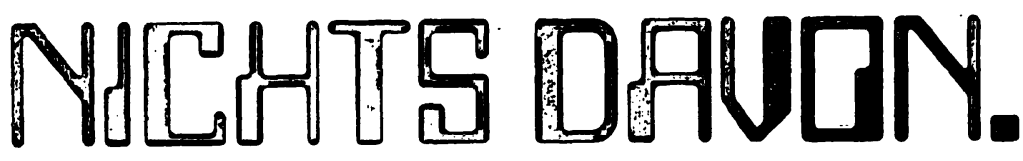

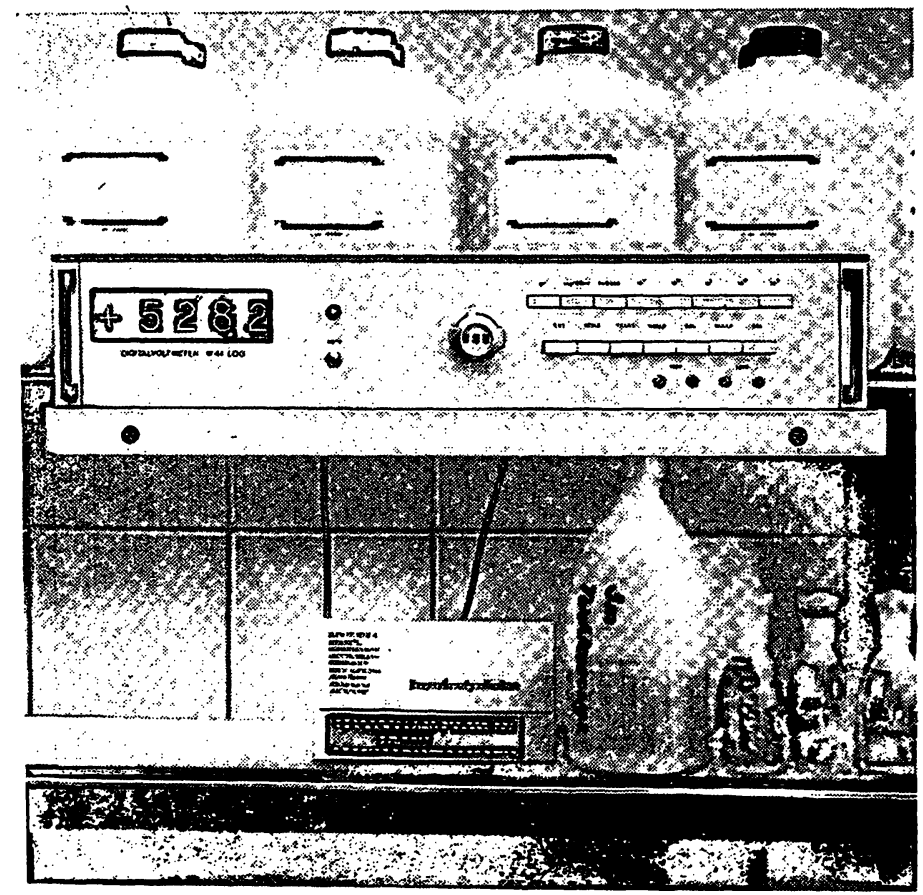

reichen messen. Die Werte von Transmission, Extinktion, Konzentration.

2. Fehler vermeiden. Beim Ablesen, beim Rechnen, beim Schreiben.

3. Und Ihre Arbeit vereinfachen. Durch automatisches Auswerten von Massenanalysen.

Genug Eigenschaften, die Ihnen nicht egal sein sollten.

Informieren Sie sich. Schreiben Siean: Hartmann \& Braun Digital GmbH 1 Berlin 12 - Bismarckstraße 107 - Telefon (0311) $31131 \cdot$ Telex 1-83783

Es gibt genug Labors, die genug Mitarbeiter für Routinearbeiten haben. Wie sieht das bei Ihnen aus? Wir wollen Ihnen sagen, wie Sie bei Ihrer Arbeit in der klinisch-chemischen Analytik Zeitund Aufwand sparenkönnen. Damit Sie und Ihre besten Mitarbeiter sichanspruchsvolleren Aufgaben zuwenden können.

Arbeiten Sie mit unserem DigitalVoltmeter W 44 log.

Sie können damit:

1. In drei Anwendungsbe- 